\title{
Diagnosis of Atrial Fibrillation Using Electrograms from Chronic Leads: Evaluation of Computer Algorithms
}

\author{
JANICE JENKINS, ${ }^{*}$ KI HONG NOH, ${ }^{*}$ ALAIN GUEZENNEC, THOMAS BUMP, ** \\ and ROBERT ARZBAECHER \\ Pritzker Institute of Medical Engineering, IIT Center, Chicago, IL; *The University of Michigan, \\ Ann Arbor, MI; and **The University of Chicago, Chicago, IL
}

\begin{abstract}
JENKINS, J., ET AL.: Diagnosis of atrial fibrillation using electrograms from chronic leads: evaluation of computer algorithms. This study compares the performance of three detection algorithms for the recognition of atrial fibrillation in chronic pacing leads. Multiple serial recordings were obtained of wideband and filtered electrograms from chronic atrial and ventricular leads in dogs for a period up to 55 days following implantation. Each dog was recorded in sinus rhythm and induced atrial fibrillation. Four days were chosen for processing: The day of implantation and a day in the first, second or third, and fifth weeks. Three signal processing methods were assessed for performance in detection of atrial fibrillation: software recognition of rate with automatic threshold control, amplitude distribution, and frequency spectral analysis. A software trigger for rate determination was adjusted to thresholds of 10,20 , and $30 \%$ of maximum baseline-to-peak amplitude. At $10 \%$, a rate boundary anywhere between 420 and 560 beats per minute (bpm) perfectly separated atrial fibrillation from sinus rhythm even though atrial electrograms were contaminated with large QRS deflections and double-sensing was present. At $20 \%$ and $30 \%$, a rate boundary around $300 \mathrm{bpm}$ could be used, but sensitivity and specificity were reduced to $90 \%$. In amplitude distribution analysis, a percent of time within a baseline window provided perfect separation of atrial fibrillation from sinus rhythm. In all cases, the signal was within this window less than $43 \%$ of the time in atrial fibrillation, and more than $43 \%$ in sinus rhythm. In spectral analysis, frequency bands were examined for power content. In the 6 to $30 \mathrm{~Hz}$ band atrial fibrillation contained the greater power. Choosing $58 \%$ of total power as a discriminant, sensitivity and specificity of atrial fibrillation detection were $100 \%$ and 95\% respectively. (PACE, Vol. 11, May 1988)
\end{abstract}

atrial fibrillation, atrial rate analysis, amplitude distribution analysis, frequency spectral analysis

\section{Introduction}

The accurate diagnosis of atrial fibrillation is an important task in the design of antitachycardia devices. Atrial fibrillation can occur in patients who also have supraventricular tachycardia or ventricular tachycardia, and atrial fibrillation can

This work was supported in part by National Institutes of Health grants HL 35554, HL 32131, and National Science Foundation grant ECS-8351215.

Address for reprints: Janice M. Jenkins, Ph.D., Department of Electrical Engineering and Computer Science, The University of Michigan, Ann Arbor, MI 48109-2122.

Received October 23, 1987; accepted December 3, 1987. confound devices which are used to control these other arrhythmias. In addition, it is possible that future devices will be used which are specifically designed to control atrial fibrillation. ${ }^{1}$

Existing algorithms for automatic diagnosis of rhythm by devices are mostly based on rate in either chamber, or change of rate. ${ }^{2,3,4}$ For these algorithms to detect atrial fibrillation, the atrial electrogram during fibrillation must have discrete deflections of sufficient amplitude to trigger an electronic counting circuit. Unfortunately, sensing difficulties from chronic atrial leads are com$\mathrm{mon}^{5}$ and the atrial rate during atrial fibrillation can be grossly undercounted by a circuit whose sensing characteristics are set for sinus rhythm. 
lation from atrial electrograms: atrial rate, amplitude distribution, and spectral analysis. All three methods achieved reasonable accuracy in distinguishing atrial fibrillation from sinus rhythm. Atrial rate and amplitude density analysis both completely separated episodes of sinus rhythm from episodes of atrial fibrillation. Spectral analysis completely separated episodes of the two rhythms with the exception of one episode of sinus rhythm.

Slocum et al. ${ }^{13}$ have previously compared similar algorithms for differentiating sinus rhythm from atrial fibrillation. These authors found that amplitude distribution was the most reliable technique. Our study differs from theirs in that we recorded both sinus rhythm and atrial fibrillation in each subject, and we used chronically implanted permanent pacemaker leads and recorded electrograms not only on the day of implantation but also on subsequent days. Also, our data base included atrial electrograms with sizable QRS artifacts. In our study, the performance of the three algorithms seem particularly robust when one considers the marked variability among different electrograms. The algorithms were also robust in another sense. Each algorithm maintained its accuracy despite different choices in feature measurement. For example, the rate algorithm separated atrial fibrillation from sinus rhythm (with at least 90\% sensitivity and 90\% specificity) at all three defined sensing thresholds. The amplitude distribution algorithm separated atrial fibrillation from sinus rhythm at all four defined window sizes. In this small training set a $100 \%$ separation was possible, but some data points were close to the $43 \%$ boundary for both sinus rhythm and atrial fibrillation. In a larger population we would expect some overlap and less than perfect result. The power density spectral algorithm separated atrial fibrillation from sinus rhythm at four different defined frequency bands. Thus, these algorithms do not demand enormous precision in feature extraction.

The three algorithms also permit flexibility in decision rules. For each algorithm, the boundary separating atrial fibrillation from normal sinus rhythm could be varied over a wide range of values with maintained accuracy.

All three algorithms could be implemented in implantable devices. The rate algorithm with its software-determined sensing threshold and the amplitude distribution algorithm both could be implemented on the kind of microprocessors which even now are finding their way into implantable devices. The power density spectral method would require chips with special purpose architecture; these are technologically feasible. However, the power density spectral algorithm separates these rhythms using unfiltered data. It is unreasonable to expect that pacemaker sensing amplifiers will ever use unfiltered signals, since enormous difficulties would arise from baseline wander, electrode polarization, and low frequency artifacts including myocardial repolarization.

Our study had the following limitations. First, our study was performed in dogs, because we could not get serial high-fidelity recordings from implanted electrodes in humans, and it is not clear whether our findings can be extrapolated to humans. Second our decision boundaries were produced through analysis of the entire data set. Decision boundaries and criteria for detection were determined retrospectively and results reflect only the small population of this training set. While we were able to get a sense of the robustness of each of the three algorithms, evaluation of the true sensitivity and specificity will have to be tested prospectively on a larger test set. No analysis of electrograms was made during exercise or motion since the dogs were recorded while anesthetized. Positional changes were present though due to the extended period of this chronic study. Finally, we applied the algorithms only to two widely different arrhythmias, sinus rhythm and atrial fibrillation. Further work should be done in which supraventricular tachycardia and atrial flutter are included in the data set.

\section{References}

1. Arzbaecher R, Bump T, Munkenbeck F, et al. An algorithm for automatic infusion of procainamide in acute management of paroxysmal atrial fibrillation. Computers in Cardiology. 1984, p. 57.
2. Jenkins J, Bump T, Glick K, et al. Automated recognition of tachycardias from electrograms: Decision rules for diagnosis. Computers in Cardiology. 1983, p. 93. 
3. Jenkins J, Bump T, Munkenbeck F, et al. Tachycardia detection in implantable antitachycardia devices. PACE 1984; 7:1273.

4. Arzbaecher R, Bump T, Jenkins J, et al. Automated tachycardia recognition. PACE 1984; 7:541.

5. Echt D. Potential hazards of implanted devices for the electrical control of tachyarrhythmia. PACE 1984; 7:580.

6. Furman S, Fisher JD, Pannizzo F. Necessity of signal processing in tachycardia detection. In SS Barold and J Mugica (Eds): The Third Decade of Cardiac Pacing. Mount Kisco, New York, Futura Publishing Co., 1982, p. 265.

7. DeCaprio V, Hurzeler P, Furman S. A comparison of unipolar and bipolar electrograms for cardiac pacemaker sensing. Circulation 1977; 56:750.

8. Kleinert M, Elmquist H, Strandberg H. Spectral properties of atrial and ventricular endocardial signals. PACE 1979; 2:11.

9. Parsonnet V, Myers GH, Kresh YM. Characteristics of intracardiac electrograms II. Atrial endocardial electrograms. PACE 1980; 3:406.

10. Morkrid L, Ohm OJ, Engedal H. Time domain and spectral analysis of electrograms in man during regular ventricular activity and ventricular fibrillation. IEEE Trans Biomed Eng 1984; BME-31:350.

11. Goldreyer BN, Almquist CK, Beck RC, et al. Waveform and frequency analysis of unipolar, bipolar, and orthogonal atrial electrograms. (Abstr) PACE 1986; 6:283.
12. Parker B. Fourier Analysis of electrograms. PACE 1979; 2:246.

13. Slocum J, Sahakian A, Swiryn S. Characteristics of atrial fibrillation. Computer Interpretation of the Electrocardiogram XI. New York, New York, Engineering Foundation, 1986, p. 102.

14. Mirowski M, Mower MM, Reid P, et al. The automatic implantable defibrillator. PACE 1982; 5:384.

15. Lin D, Jenkins J, Jadvar H, DiCarlo L. Analysis of time and frequency domain patterns of endocardial electrograms to distinguish ventricular tachycardia from sinus rhythm. Computers in Cardiology 1986, p. 171.

16. Ahmed N, Natarajan T. Discrete-Time Signals and Systems. Reston, Virginia, Reston Publishing Co., Inc., 1983.

17. Richards PI. Computing reliable power spectra IEEE Spectrum 1967; 4:83.

18. Bingham C, Godfrey MD, Tukey JW. Modern techniques of power spectrum estimation. IEEE Trans Audio Electroacoustics 1967; AU-15:56.

19. Welch PD. The use of fast Fourier transform for the estimation of power spectra: A method based on time averaging over short, modified periodograms. IEEE Trans Audio Electroacoustics 1967; AU-15:70.

20. Kerr CR, Mason MA. Amplitude of atrial electrical activity during sinus rhythm and during atrial fibrillation. PACE 1985; 8:348.

INTERNATIONAL POST-GRADUATE COURSE

CARDIAC PACING COMPLICATIONS AND RATE RESPONSIVE PACING

\author{
JUNE 11-13, 1988 \\ Dubrovnik, Yugoslavia
}

For Information Contact:

Milan Djordjevic, M.D.

Institute of Surgery

Pacemaker Center

Visegradska 26

Belgrade, Yugoslavia 